\title{
Changes in daily measures of blood pressure and heart rate improve weight-based detection of heart failure deterioration in patients on telemonitoring
}

Citation for published version (APA):

Joshi, R., \& Cuba Gyllensten, I. (2019). Changes in daily measures of blood pressure and heart rate improve weight-based detection of heart failure deterioration in patients on telemonitoring. IEEE Journal of Biomedical and Health Informatics, 23(3), 1041-1048. [8412489]. https://doi.org/10.1109/JBHI.2018.2856916

DOI:

10.1109/JBHI.2018.2856916

Document status and date:

Published: 01/05/2019

Document Version:

Accepted manuscript including changes made at the peer-review stage

Please check the document version of this publication:

- A submitted manuscript is the version of the article upon submission and before peer-review. There can be important differences between the submitted version and the official published version of record. People interested in the research are advised to contact the author for the final version of the publication, or visit the $\mathrm{DOI}$ to the publisher's website.

- The final author version and the galley proof are versions of the publication after peer review.

- The final published version features the final layout of the paper including the volume, issue and page numbers.

Link to publication

\footnotetext{
General rights

- You may freely distribute the URL identifying the publication in the public portal. follow below link for the End User Agreement:

www.tue.nl/taverne

\section{Take down policy}

If you believe that this document breaches copyright please contact us at:

openaccess@tue.nl

providing details and we will investigate your claim.
}

Copyright and moral rights for the publications made accessible in the public portal are retained by the authors and/or other copyright owners and it is a condition of accessing publications that users recognise and abide by the legal requirements associated with these rights.

- Users may download and print one copy of any publication from the public portal for the purpose of private study or research.

- You may not further distribute the material or use it for any profit-making activity or commercial gain

If the publication is distributed under the terms of Article 25fa of the Dutch Copyright Act, indicated by the "Taverne" license above, please 


\title{
Changes in Daily Measures of Blood Pressure and Heart Rate Improve Weight-based Detection of Heart Failure Deterioration in Patients on Telemonitoring
}

\author{
Rohan Joshi and Illapha Cuba Gyllensten
}

\begin{abstract}
Blood pressure (BP) and heart rate (HR) are often captured in conjunction with weight in telemonitoring systems, but the additional prognostic potential of daily measurements of $B P$ and $H R$ in providing information on upcoming hospitalizations for worsening heart failure (HFH) have not been explored thoroughly. We retrospectively analyzed 267 daily hometelemonitored heart failure (HF) subjects. We extracted those episodes of HFHs that had sufficient data entries in the days leading up to hospitalization and tested the prognostic potential of 48 trend features based on weight, systolic BP, diastolic BP, pulse pressure (PP) and HR with a Naïve Bayesian model. The single best-performing trend feature - with a cross-validated estimate of 0.64 for the area under the curve (AUC) with a standard deviation (SD) of 0.01 - is based on a 2-day weight trend. The best multivariate feature set (cross-validated $\mathrm{AUC}=0.70, \mathrm{SD}=0.01$ ) comprises of 2-day trend features based on weight, systolic BP, and HR. There were large variations in the weight trends preceding hospitalizations and weight-change alone had a modest predictive ability. Readily interpretable features capturing trends in BP and HR provided additional prognostic information and can be used for improving classification.
\end{abstract}

Index Terms-Telemedicine, Blood pressure, Heart rate, Heart Failure, Decompensation.

\section{INTRODUCTION}

$\mathrm{T}$ HE path of deterioration from "stable" Heart Failure (HF) to acute decompensated HF is often preceded by a period of worsening hemodynamics, spanning several days or even weeks before manifesting itself acutely. Therefore, theoretically, a window of opportunity presents itself during which decompensation might be reflected in physiological signals and thus could be acted upon. Home-telemonitoring could be used as a tool to allow monitoring of the vital parameters of patients and provide education and follow-up for them. Together with promoting medication adherence and optimized self-care behaviors, this could not only improve patient outcomes but also reduce costs and the burden on the

This paragraph of the first footnote will contain the date on which you submitted your paper for review. It will also contain support information, including sponsor and financial support acknowledgment. For example, "This work was supported in part by the U.S. Department of Commerce under Grant BS123456." healthcare systems by keeping the patients stable.

Despite the many purported advantages of such an integrated system, the evidence for such implementation with noninvasive measures of weight, blood pressure, and heart rate is conflicting. Although most meta-analyses of HF telemonitoring trials have concluded that in comparison with usual care, telemonitoring reduces all-cause hospitalizations, hospitalizations for worsening heart failure (HFH), and allcause mortality, different conclusions have been drawn from recent larger trials. A cohesive interpretation of the findings is complicated by inter-trial differences among the interventions [1]-[9]. Better selection of telemonitoring service model, integration of multi-disciplinary team and improving the use and interpretation of the data captured by the system should improve this.

In addition to observations by medical personnel, telemonitoring also provides the possibility of automatically analyzing the physiological signals measured at the patient's home in order to predict an increased risk of decompensation. In particular, some episodes of decompensated heart failure are preceded by an increase in body weight — as a result of water retention-and this finding has been exploited as part of developing predictive models that "recognize" deterioration [10]-[14]. While the prognostic potential of an increase in weight before heart failure has been established, its sensitivity is poor [11], [13]-[15]. Existing studies that have used weightbased features have employed a range of analyses and different techniques to evaluate algorithms, often using a small number of hospitalized patients. Therefore, comparisons between various studies are difficult and, as a result, many issues remain unresolved. Improved detection of deterioration could help telemonitoring nurses to more effectively direct their attention to patients at higher risk and provide better care through telemonitoring if properly integrated [16].

This study aimed to determine if there was any additional value of including trends in blood pressure (BP) and heart rate (HR),

R. Joshi, is with the Department of Fertility, Pregnancy and Parenting Solutions, Philips Research, Eindhoven, and the Department of Industrial Design, Eindhoven University of Technology, Eindhoven.

I. Cuba Gyllensten is with the Department of Personal Health Solutions, Philips Research, Eindhoven, and the Department of Electrical Engineering, Eindhoven University of Technology, Eindhoven. (e-mail: illapha.cuba.gyllensten@philips.com). 
which are often captured in conjunction with weight, into the detection of deterioration.

\section{METHODS}

\section{A. Patient Population}

The data used for this study was retroactively collected from the Trans-European Network-Home-Care Management System (TEN-HMS) [17] and from an ongoing telemonitoring service in Hull, UK (Motiva-Hull) in which patients with a recent admission for worsening HF could be enrolled. In both studies the same system was used to log weight and blood pressure. Amongst other criteria, at the time of enrolment, the TEN-HMS study excluded subjects with a left ventricular ejection fraction (LVEF) greater than $40 \%$. With the intention of homogenizing the two databases, we excluded those patients in the MotivaHull database who, upon enrollment, presented with an LVEF greater than $40 \%$ and those with simultaneously missing records for both LVEF and N-terminal pro-brain natriuretic peptide (NT-proBNP). A total of 267 patients, 167 from the TEN-HMS database and 100 from the Motiva-Hull database, fulfilled these criteria and constituted the available dataset for analysis. Table I characterizes the baseline demographics, medications, presence/absence of implanted cardiac pacing device (ICD/CRT/pacemaker), and the values for EF, NTproBNP, and electrolytes for the this combined patient population. The "\% missingness" column refers to the percentage of subjects for whom values were unavailable at the time of enrollment.

TABLE I

BASELINE CHARACTERISTICS OF SUBJECTS FROM THE TWO DATABASES

\begin{tabular}{|c|c|c|}
\hline Characteristic & Baseline value & $\%$ missingness \\
\hline No. of subjects & 267 & - \\
\hline Mean age, years (SD) & $68.4(12.5)$ & 0 \\
\hline$\%$ Female & 19.1 & 0 \\
\hline Mean body mass index (SD) & $26.3(5.0)$ & 21 \\
\hline Mean LVEF, \% (SD) & $26.1(7.9)$ & 20.2 \\
\hline $\begin{array}{l}\text { NT-proBNP } \quad(\mathrm{pg} / \mathrm{ml}), \quad \text { Median } \\
\text { [IQR] }\end{array}$ & $\begin{array}{l}3120 \\
{[1399-6051]}\end{array}$ & 14.2 \\
\hline $\begin{array}{l}\text { Serum creatinine }(\mu \mathrm{mol} / \mathrm{l}) \text {, Mean } \\
\text { (SD) }\end{array}$ & $134.8(60.4)$ & 7.9 \\
\hline Serum urea $(\mathrm{mg} / \mathrm{dl})$, Mean (SD) & $12.1(14.5)$ & 7.9 \\
\hline $\begin{array}{l}\text { Serum sodium }(\mathrm{mmol} / \mathrm{l}) \text {, Mean } \\
\text { (SD) }\end{array}$ & $137.3(4.4)$ & 8.2 \\
\hline $\begin{array}{l}\text { Serum potassium }(\mathrm{mmol} / \mathrm{l}) \text {, Mean } \\
\text { (SD) }\end{array}$ & $4.4(0.50)$ & 8.6 \\
\hline \multicolumn{3}{|l|}{ Treatment at baseline } \\
\hline Diuretics (\%) & 95.9 & - \\
\hline ACE inhibitors/ARBs (\%) & 83.5 & - \\
\hline Beta-blockers (\%) & 62.9 & - \\
\hline Vasodilators (\%) & 27.0 & - \\
\hline Digoxin (\%) & 37.4 & - \\
\hline ICD/CRT/pacemaker (\%) & 13.1 & - \\
\hline
\end{tabular}

$\mathrm{SD}=$ standard deviation, $\mathrm{IQR}=$ interquartile range, $\mathrm{ACE}=$ angiotensinconverting enzyme, $\mathrm{ARB}=$ angiotensin-receptor blocker.

All subjects were required to use a telemonitoring device installed in their homes for recording their weight, BP, and HR every day, at a fixed time. Each instance of measurement resulted in a single value for weight, systolic BP, diastolic BP, and HR. For our study, in the case of multiple daily measurements, only the first measurement of the day was retained, while data corresponding to the days with no recordings were imputed with linear interpolation between the closest recordings.

Morbidity and mortality were high in the patient population under study, although lower than the patients who were invited but declined telemonitoring [18] or with patients matched for propensity scores [19]. For the study population, the KaplanMeier estimates at two years were $25 \%$ for all-cause-mortality and $50 \%$ for hospitalization due to worsening HF. Furthermore, after approximately three years, nearly $80 \%$ of the subjects had either died or had at least one episode of HFH. At three years, all subjects had at least one hospitalization, if non-HF related hospitalizations were also included.

With regard to the number of HFHs, in the combined dataset of 267 patients, 111 presented with $256 \mathrm{HFHs}$ during the period of monitoring (until death/censoring). The remaining 156 subjects did not suffer from any episode of HFH (or die from $\mathrm{HF}$ ) during the period of monitoring. No significant differences ( $p>0.05$ ) were present in the baseline characteristics between these two groups with the exceptions of NT-proBNP and serum sodium values, which were higher and lower respectively in the 111 subjects who got hospitalized. The lower serum sodium levels were perhaps due to greater water retention despite titrated diuretic therapy [21].

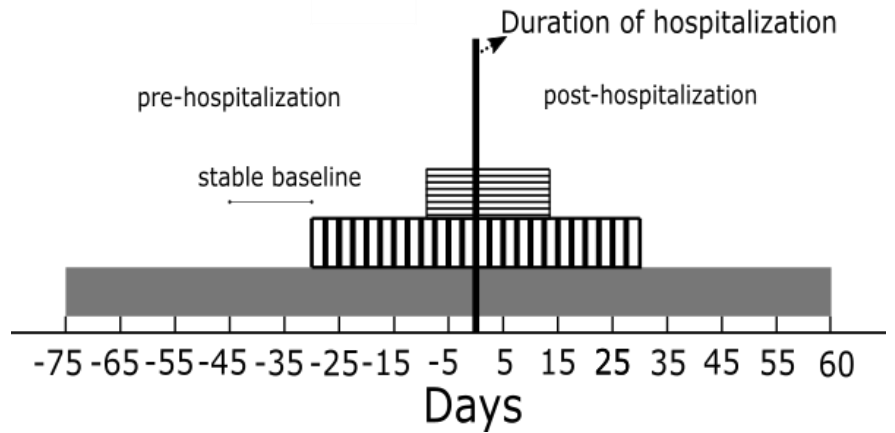

Fig. 1. Visualizing the selection criteria that were applied to the cases of HFHs and controls. The black line represents the potentially multi-day admission to the hospital, during which no telemonitoring data is gathered, and the subsequent discharge. The opaque section represent the event-free days (see Methods for details), while the sections corresponding to vertical and horizontal stripes, respectively represent the periods with a requirement of at least $50 \%$ data entries. The period between 30-45 days before hospitalization served as a stable period used in the calculation of multiple features. All cases that satisfied the pre-admission criteria were used for analysis while those that satisfied the post-discharge criteria as well were used for visualizing the effect of hospitalization on the physiological signals.

\section{B. Selecting Cases of HFHs and Controls}

Since not all episodes of HFHs have sufficient telemonitoring data in the days preceding hospitalization, not all cases of HFHs can be used for analyses. For analysis, data from the 30-day window preceding an HFH is vital, since this is the period during which physiological changes are expected to manifest [10], [20]. Therefore we retained only those cases of HFHs that had at least $50 \%$ data entries (empirical choice) in the 30-day and the eight-day windows preceding hospitalization. While data sufficiency in the days preceding 
$\mathrm{HFH}$ is important, it is also necessary to ensure that the data is solely reflective of the impending HFH and free from the influence of any past "events" experienced by the patient. To avoid such "contamination," only those instances of HFHs were retained where the 75 days preceding HFH were free of "events," defined as enrollment into the study or discharge from an earlier HFH. The mean values of the physiological signals between the 30th day and the 45th-day preceding HFH served as a "stable baseline" reference for every subject.

To determine whether the physiological variations before HFH are distinctive, they need to be contrasted against naturally occurring changes in physiology, hereafter referred to as control cases. To obtain the time-series corresponding to control cases, we used those subjects who did not experience an episode of HF leading to hospitalization (or death) during the period of monitoring (until censoring/death). Data sufficiency criteria, identical to those used for the cases of HFHs were applied at five random points (control "events") on the time-series data of these subjects. Here, too, a "control-case" had to be at least 75 days away from enrollment into the study. These data, corresponding to both the cases of HFHs and the controls, were used to generate and test features.

Previously researched algorithms that have attempted to capture weight-gain preceding HF have had limited sensitivity. We sought to explain these results by quantifying the episodes of HF that do exhibit weight-gain preceding hospitalization. Weight-trends were stratified as "uptrend," "downtrend" or "no trend," based on whether the mean weight in the ten days preceding $\mathrm{HFH}$ increased by, decreased by, or stayed within one kilogram of the stable baseline value.

While the data preceding $\mathrm{HFH}$ is relevant for analyzing the predictive potential of the different physiological signals, the post-discharge data, reflective of treatment, can hold useful information on the return to baseline. To observe this effect, we applied certain data sufficiency criteria to the stream of data following discharge from HFH. These criteria required at least $50 \%$ data in the ten-day and the 30-day window post-discharge, along with an absence of "events" in the 60 days post-discharge. Here, "events" refers to HF leading to hospitalization or death. Fig. 1 shows the selection criteria that were applied to the cases of HFHs and controls.

\section{Feature Generation}

A battery of 48 features was developed for evaluating the prognostic potential of time-series data of weight (16 features), systolic BP (eight features), diastolic BP (eight features), PP (eight features), and HR (eight features) for identifying periods of deteriorating HF. All features were developed from three "families of features."

The first feature-family was the rule-of-thumb algorithm that was motivated by the guideline thresholds of two $\mathrm{kg}$ weight gain in two to three days or two percent weight gain in two days [11]. Such features were intended to capture acute discriminative changes in weight. Six weight-based features corresponding to one-, two-, and three-day differences in absolute and percentage weight change were employed. Since evidence of an increase in weight starting up to a month before
HF deterioration was available [10], we also developed features corresponding to the differences (absolute and percentage) between the mean weights measured over a week, a fortnight, and a month with respect to stable baseline values giving rise to six features.

For the systolic BP, diastolic BP, PP, and HR, four features apiece were developed to capture the absolute differences between the mean values measured over the past week, the penultimate week (this was an empirical choice motivated by observing a bump in the population-level systolic BP trend in the week penultimate to $\mathrm{HFH}$ ), the past fortnight, and the past month with respect to stable baseline values.

The second feature-family used the moving average convergence divergence (MACD) algorithm, i.e., the difference between the mean of short-term data (short-term window size (STWS)) and a long-term average (long-term window size (LTWS)). The use of this feature-family, which was motivated by the work of Zhang et al. [15], was employed for weight, BP (systolic, diastolic and pulse pressure), and HR using LTWSs of 20 and 40 days respectively, along with an STWS of five days. In effect, the MACD algorithm acts like the combination of a differential operator and a low pass filter and is intended to capture fast changes w.r.t. to slower-changing trends.

The third feature-family was based on the standard score (SS), which was defined as:

$$
S S(\mathrm{t})=\frac{x(t)-\mu}{\sigma}
$$

where, $x(t)$ is the value of the time-series signal at time " $t$ " while " $\mu$ " and " $\sigma$ " are the mean and standard deviation, respectively, calculated from a predefined length of past values. SS-based features were employed for weight, BP (systolic, diastolic and pulse pressure), and HR using sample lengths of 20 and 40 days respectively. The SS-based features were designed to capture changes in a subject's physiological signals that differ significantly from the naturally exhibited variation of an individual. Both, the MACD and the SS-based features used the original, uninterpolated data.

\section{Classification Evaluation and Statistics}

While decompensation is believed to be reflected in physiological changes, the precise point in time when such deterioration peaks before requiring hospitalization are unknown. Therefore, the analysis was carried out using different lengths of data (two, five, eight, and twelve-day windows) from the subject's past measurements. In this scenario, the entire "window of data" of a subject is considered to correspond to "HFH" or a "control," as the case may be and is fed to the classifier. For example, a five-day window would yield 5 data points corresponding to HFH or of control. It should be noted that constructing all features required data from the past. So to calculate a feature value on the $12^{\text {th }}$ day prior to $\mathrm{HFH}$, data from before the $12^{\text {th }}$ day is needed. This is the reason why we applied the criteria of $50 \%$ data availability in the 30 day period before $\mathrm{HFH}$.

The Naïve Bayes classifier was used to distinguish daily data belonging to the period leading up to a HFH from control periods. The class priors were defined to be uniform while 
continuous data were represented by estimating non-parametric densities using multiple kernels of the normal distribution. The predictive strength of the classifier was evaluated by calculating the area under the curve (AUC) from the concatenated left out folds of a fivefold cross-validation (CV) stratified by HFH and control data, carried out five times to provide an average AUC estimate and estimates of its dispersion (standard deviation).

While the AUC measures the predictive strength of the classifier, it does not explain how changes in the individual features affect the probability of decompensation. Thus, to make the classification process easier to interpret, the calculated probabilities of decompensation were extracted for different values of the individual features. The means and standard deviations of these values were then calculated over the different runs of the $\mathrm{CV}$ process for a model (chosen to serve as an example) and plotted individually for each feature.

Differences in the baseline characteristics between the cohorts were evaluated with Welch's test for the continuous variables and with the chi-squared test for categorical variables, a nominal p-value of 0.05 was assumed throughout. All models were developed and evaluated in a MATLAB environment (MATLAB R2014 MathWorks).

\section{RESULTS}

\section{A. Characteristics of the HFH and the Control Subjects}

Upon implementing the selection criteria described in section 2.2, 50 instances of HFHs and 112 of controls were found suitable for quantitative analysis. These instances originated from 40 and 63 patients from the hospitalized and the control group respectively, baseline characteristics of which are shown in Table II. The large decrease in the number of HFHs available for analysis, from 256 to 50 , is primarily attributable to most episodes of HFHs occurring within 75 days of discharge from a previous $\mathrm{HFH}$ or from enrolment into the study. This is not entirely surprising since the burden of disease in these patients was high.

TABLE II

BASELINE CHARACTERISTICS OF THE HFH AND CONTROL COHORTS.

\begin{tabular}{llll}
\hline \hline Characteristic & HFH-cohort & $\begin{array}{l}\text { Control- } \\
\text { cohort }\end{array}$ & $\begin{array}{l}\text { p- } \\
\text { value }\end{array}$ \\
\hline No. of subjects & 40 & 63 & - \\
No. of cases & 50 & 112 & 0.24 \\
Mean age, years (SD) & $66.2(11.2)$ & $62.5(15.5)$ & 0.92 \\
\% Female & 17.5 & 16.7 & 0.06 \\
Mean body mass index (SD) & $28.5(5.9)$ & $26.20(4.14)$ & 0.65 \\
Mean LVEF, \% (SD) & $26(7.3)$ & $25.2(6.84)$ & 0.18 \\
NT-proBNP (pg/ml), Median & 2921 & 2545 & 0.71 \\
[IQR] creatinine ( $\mu$ mol/l), & $130.4(55.3)$ & {$[972-5545]$} & \\
Serum & & & \\
Mean (SD) & & $137.2(94.42)$ & 0.66 \\
Serum urea (mg/dl), Mean (SD) & $11.2(6.8)$ & $10.4(9.38)$ & 0.84 \\
Serum sodium (mmol/l), Mean & $137.6(3.4)$ & $137.8(4.70)$ & 0.67 \\
(SD) & & & \\
Serum potassium (mmol/l) & $4.4(0.5)$ & $4.4(0.55)$ & 0.24 \\
Mean (SD) & & & \\
\hline \hline
\end{tabular}

p-values for the continuous variables are estimated using Welch's student t-test (assuming equal means, unknown and unequal variance).

\section{B. Physiological Trends Preceding HFH and Following Discharge}

While 50 episodes of HFHs had fulfilled the pre-admission criteria, only 44 episodes of HFHs remained after applying the post-discharge criteria. These were used for visualizing the influence of hospitalization on physiological signals. Out of the five physiological signals considered, only weight changed visibly in the days leading up to $\mathrm{HFH}$ and after discharge. A closer analysis of the weight trends, based on stratifying trends leading up to hospitalization (see Fig. 2) shows that $41 \%$ of decompensations do not exhibit any increase in weight before HFH. In fact, weight decreased in $20 \%$ of the cases in the weeks before hospitalization with some increase, possibly edematous, in the days immediately preceding HFH.

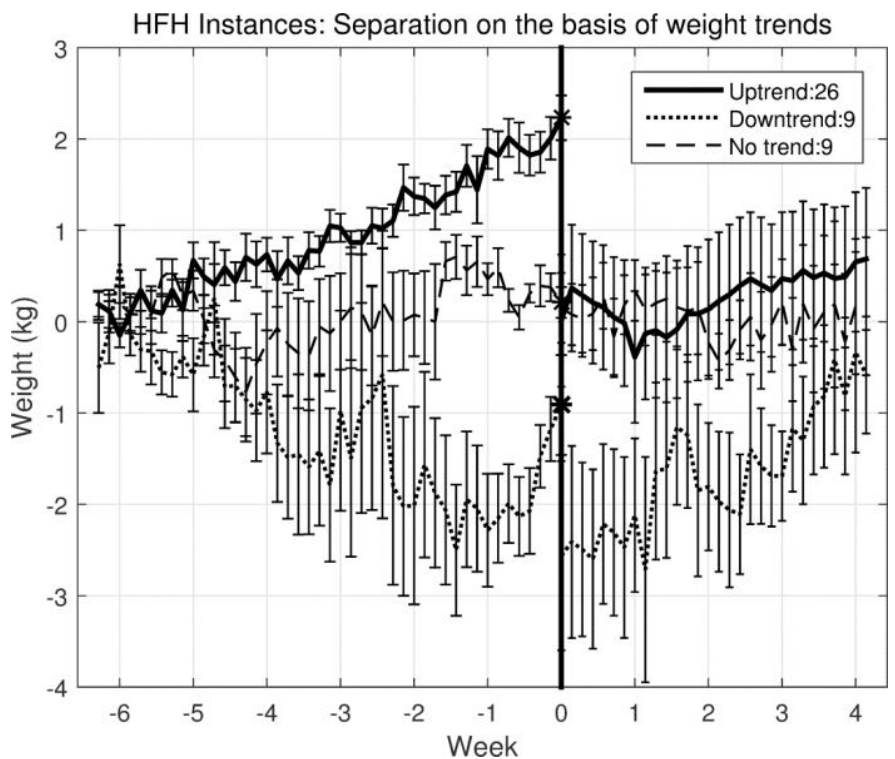

Fig. 2. Stratification of the instances of HFH by weight (stable baseline removed) showing an uptrend, a downtrend, or a flat trend in the weeks preceding HFH. These trends depict the mean population weight, and the error bars represent the standard error of the mean. The legend indicates the number of subjects in each subgroup. The thick black line at "0" represents admission to the hospital potentially lasting multiple days, during which no telemonitoring data is gathered, and the subsequent discharge.

\section{Univariate analysis}

For univariate analysis, features originating from each of the five physiological signals were individually tested on the four different window lengths of data using the Naïve Bayes classifier. The best-performing feature from each physiological category could change for different window lengths. Table III details the list of features which were best-performing for at least one window length while Fig. 3 shows the corresponding AUCs.

Interestingly, while the performance of the best weight-based feature does not change much with longer windows, the best HR-based features become more discriminatory as window length increases, in fact outperforming the best weight-based feature for a window of eight days. The performance of the BPbased features is lower than that of weight and HR-based ones and tends to decrease with increasing window-lengths. 


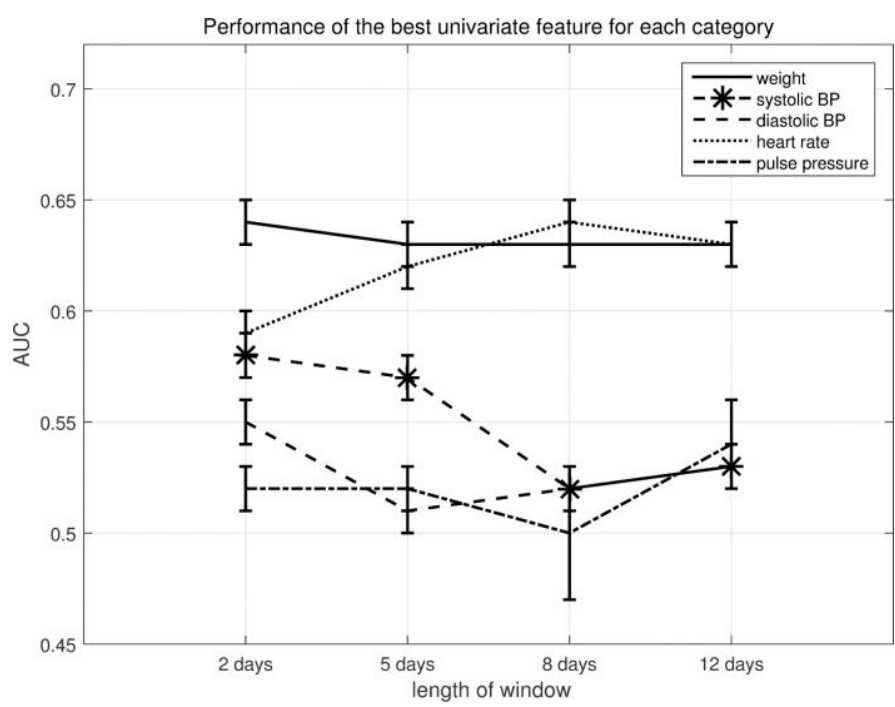

Fig. 3. This figure separately indicates the AUC values of the best performing features originating from weight, systolic BP, diastolic BP, PP and HR for window lengths of two, five, eight and 12 days. The error bars represent the standard deviation of the AUC estimates.

\section{Multivariate analysis}

For multivariate analysis, the 18 unique features listed in Table III were used in combinations of one feature up to five features at a time to examine the performance of the Naïve Bayes classifier. Fig. 4 shows the AUCs of the best-performing feature combinations for all window lengths. The performance of the classifier improves as the number of features increase, up to a combination of four features, after which it levels off or deteriorates. This pattern is common to all window lengths that were considered.
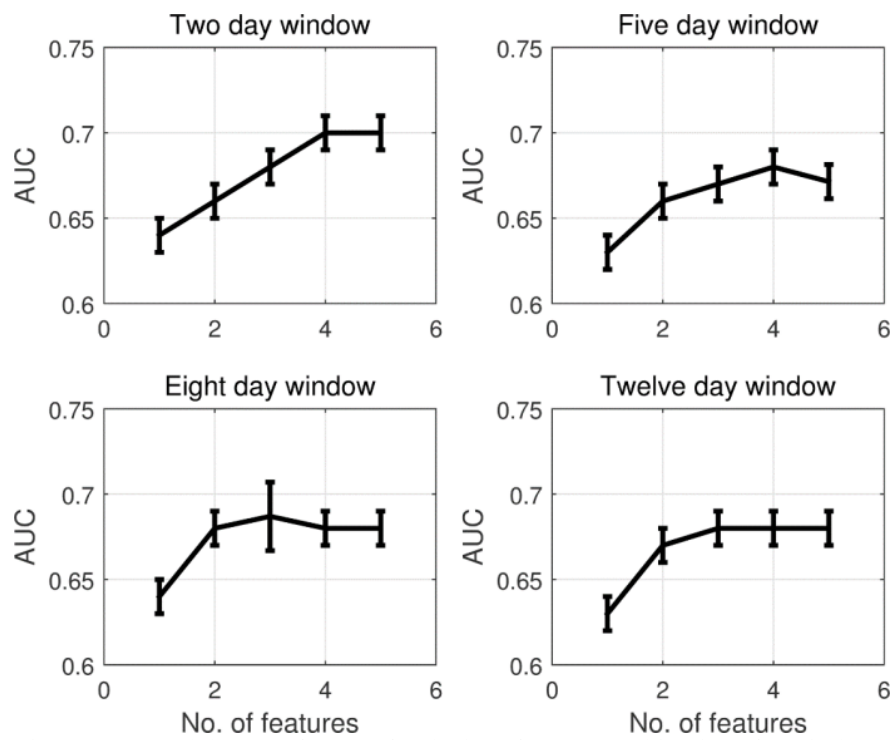

Fig. 4. AUC and the SD of AUCs for the best feature combinations for windows of length two, five, eight and twelve days. Features are taken in combinations of one (equivalent to univariate) up to five at a time. The error bars represent the standard deviation of the AUC estimates.

For a two-day window, the combination of $\mathrm{f}-4, \mathrm{f}-9, \mathrm{f}-11$ and $\mathrm{f}-12$ yields the best results, while for a five-day window, the best result is provided by the combination of $f-3, f-4, f-6$, and $f-$ 11. The weight and HR-based feature combination of $f-3, f-4$, and $\mathrm{f}-11$ give the best result for an eight-day window while $\mathrm{f}-2$, f-4, and f-11 perform best for a twelve-day window. Overall, the best performance across window size decreases with increasing the window length.

The changes in feature-values that determined classification for the top-performing features from the five-day window are shown in Fig. 5. For example, the graph corresponding to f-3 shows that the probability of decompensation increases with both increasing and decreasing values of mean weight of the past month with respect to baseline. A relatively stable weight, on the other hand, reduces the probability of decompensation (value below 0.5 ). Similarly, the graph corresponding to $f-6$ shows that lower values of systolic BP, after adjusting for the inherent variability in the systolic BP (which is what the SS measures), increase the risk of decompensation. The fact that the standard deviations are small for the classifier estimated probabilities in Fig. 5 suggests that the training was stable with little evidence of overfitting.

Since the Naïve Bayes classifier assumes independence of the features, the probability of decompensation for any subject using multivariate classification can be obtained by multiplying the probabilities of decompensation obtained from each featurevalue followed by normalization. For example, if on a certain day the probabilities of decompensation corresponding to feature values of $\mathrm{f}-3, \mathrm{f}-4, \mathrm{f}-6$ and $\mathrm{f}-11$ are $0.70,0.75,0.60$ and 0.50 , then the probability of decompensation is $0.91(=0.70 \times$ $0.75 \times 0.60 \times 0.50 /(0.70 \times 0.75 \times 0.60 \times 0.50+(1-0.7) \times(1-$ $0.75) \times(1-0.60) \times(1-0.50)))$. Interpretation should take into account the high prior probability assumed by the model $(0.50)$ that can be adjusted to baseline characteristics.

TABLE III

BEST-PERFORMING FEATURES OBTAINED FROM THE UNIVARIATE ANALYSIS

\begin{tabular}{|c|c|}
\hline $\begin{array}{l}\text { Feature } \\
\text { no. }\end{array}$ & Feature definition \\
\hline$f-1$ & $\begin{array}{l}\text { Change in mean weight of the past week with respect to (w.r.t.) } \\
\text { baseline }\end{array}$ \\
\hline f-2 & Change in mean weight of the past fortnight w.r.t. baseline \\
\hline $\mathbf{f - 3}$ & Change in mean weight of the past month w.r.t. baseline \\
\hline f-4 & $\begin{array}{l}\text { Weight-based MACD with LTWS }=40 \text { days, STWS }=5 \\
\text { days }\end{array}$ \\
\hline$f-5$ & $\begin{array}{l}\text { Systolic BP-based MACD with LTWS }=40 \text { days, STWS }=5 \\
\text { days }\end{array}$ \\
\hline f-6 & Systolic BP-based SS with sample length $=20$ days \\
\hline$f-7$ & Systolic BP-based SS with sample length $=40$ days \\
\hline$f-8$ & Change in mean diastolic BP of the past week w.r.t. baseline \\
\hline f-9 & Diastolic BP-based SS with sample length $=20$ days \\
\hline$f-10$ & Diastolic BP-based SS with sample length $=40$ days \\
\hline f-11 & Change in mean heart rate of the past month w.r.t. baseline \\
\hline f-12 & $\begin{array}{l}\text { Heart rate-based MACD with LTWS }=20 \text { days, and STWS } \\
=5 \text { days }\end{array}$ \\
\hline$f-13$ & $\begin{array}{l}\text { Heart rate-based MACD with LTWS }=40 \text { days, and STWS }= \\
5 \text { days }\end{array}$ \\
\hline$f-14$ & Heart rate-based SS with sample length $=20$ days, \\
\hline$f-15$ & Change in mean pulse pressure of the past month w.r.t. baseline \\
\hline$f-16$ & $\begin{array}{l}\text { Pulse pressure-based MACD with LTWS }=20 \text { days and STWS } \\
=5 \text { days }\end{array}$ \\
\hline$f-17$ & $\begin{array}{l}\text { Pulse pressure-based MACD with LTWS }=40 \text { days and STWS } \\
=5 \text { days }\end{array}$ \\
\hline$f-18$ & Pulse pressure-based SS with sample length $=40$ days \\
\hline
\end{tabular}

Features that contribute to the best-performing feature combinations in multivariate analysis, irrespective of window length, are highlighted in bold 


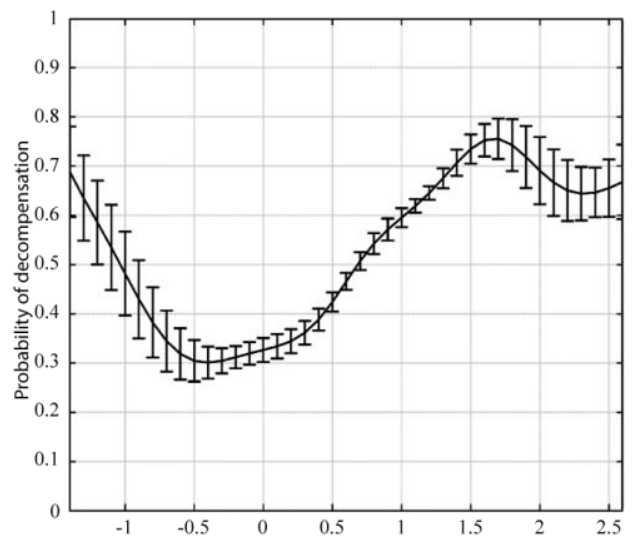

$\mathrm{f}-3$ : Change in mean weight of the past month w.r.t. baseline $(\mathrm{kg})$

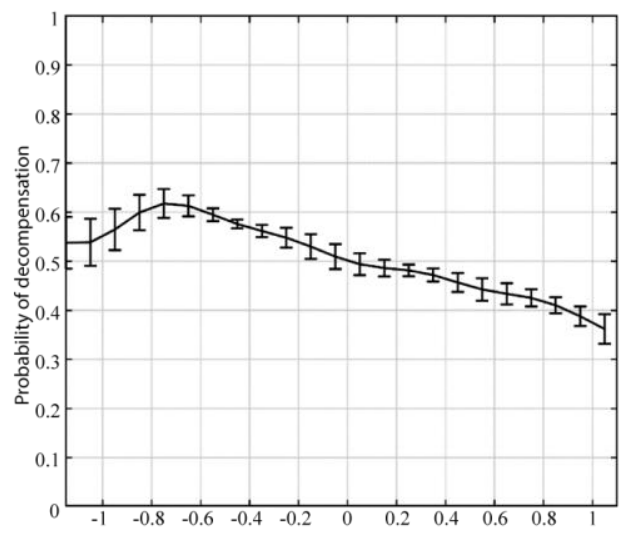

$\mathrm{f}-6$ : SS values for systolic BP using a sample length of 20 days

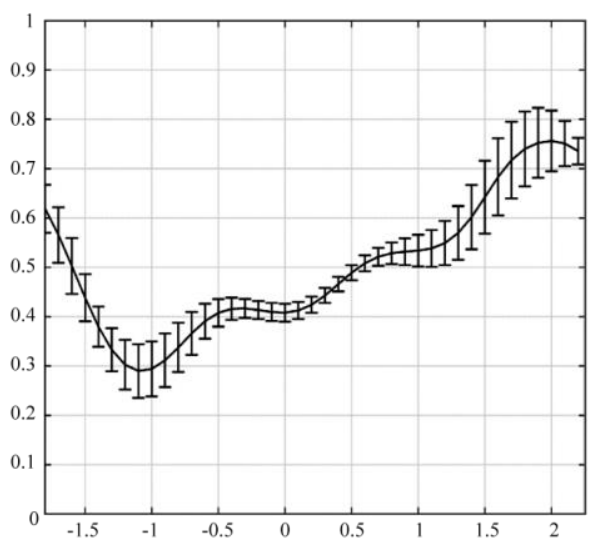

f-4: MACD values for weight with LTWS $=40$ days and STWS $=5$ days

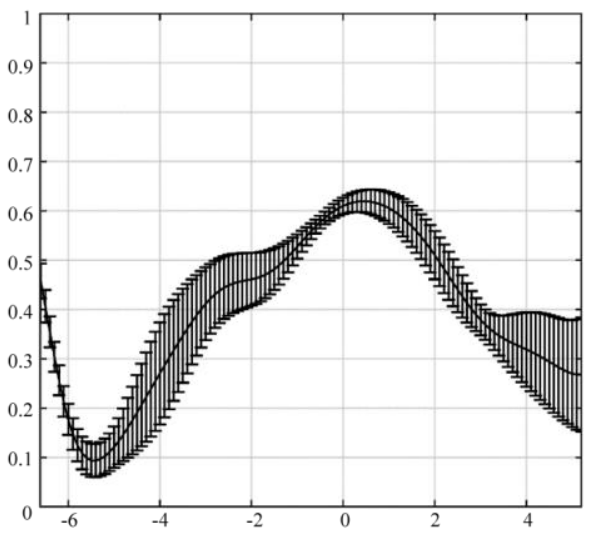

f-11: Change in mean HR of the past month w.r.t. baseline (no. of beats)

Fig. 5. Mean and standard deviation (error bars) of the classifier estimated probabilities of decompensation for the feature values of f-3, f-4, f- 6 and f-11, i.e., the features included in the top-performing five-day classifier. The $x$-axes are incremented in steps of $2 \%$. The boundaries of the $x$-axes are estimated from the $5^{\text {th }}$ and $95^{\text {th }}$ percentile estimates of the feature values, as obtained from the raw data.

\section{DISCUSSION}

Since HFHs are costly to the healthcare system and detrimental to the patient, predictive monitoring of the same is clinically important. While most studies have used weight-based features for classification, this study aimed to determine if there was any additional value of including BP and HR, parameters often captured in conjunction with weight, into the detection of deterioration.

The primary finding of this study is that medium to shortterm changes ( $\leq 8$ days) in $\mathrm{HR}$ and $\mathrm{BP}$ can add additional information to classifiers of decompensation risk in HF patients on telemonitoring. HR-based features were included in the bestperforming classifiers for all time windows under consideration, and BP-based features were included for window lengths of two and five days. Weight change features were included in all time windows, and weight was overall the most predictive feature class in the univariate analysis. This finding is similar to that of Koulaouzidis et al. with weight-based features holding the most prognostic potential. However, as opposed to their findings we find that, both, the features based on HR and on systolic BP can improve upon the prognostic potential of weight-based features alone, although this difference might be attributable to differences in the studypopulations [22]. In addition, the study of Koulaouzidis et al. studied $27 \mathrm{HFHs,}$ as opposed to 50 in this study, and was not tailored to investigate the additional potential HR and BP.

Weight gain in the week preceding HF hospitalizations occurred in the majority of the visualized cases. However, $41 \%$ of the cases had a mean weight increase of less than one kilogram, and in half of these cases, the mean weight decreased by more than one kilogram. This data highlights the heterogeneity of acute heart failure with varying presentations and shows that weight gain, although an integral part of monitoring and self-care, is insufficient as a single telemonitoring target [23]. Furthermore, the multitude of weight patterns preceding $\mathrm{HFH}$ can explain findings in the literature of low selectivity of weight-gain algorithms in predicting worsening heart failure [11], [13]-[15].

Redistribution, rather than fluid retention, has been proposed as a mechanism for decompensation [24] that could explain the lack of substantial weight gain. We further found a subgroup with a decreasing weight trend preceding HFHs. Although the reason for this weight change cannot be determined from our data, the post-discharge trend towards the baseline weight suggests that treatment following the HFHs reversed the weight loss. There are many processes that can lead to weight loss in HF [25], [26], some of which may be aggravated during the period preceding HFH, e.g., bowel wall edema and reduced intestinal blood flow causing loss of appetite and reduced nutrient uptake that could revert following treatment. If this is 
an underlying factor segmental bioimpedance of the legs, abdomen, and thorax may provide a method to distinguish and classify the same [27]. In a large study of 6933 chronic HF patients, Pockock et al. found that loss of weight in the last 12 months was an important predictor of all-cause mortality [28]. Furthermore, a small reduction in weight from admission to discharge and a post-discharge trend of increasing weight has been linked to an increase 180-day post-discharge mortality [29]. Our finding supports the importance of weight loss for $\mathrm{HFH}$ and the link with trends at presentation and discharge, which could be followed with telemonitoring.

Although neither BP nor HR presented any clear trends on a population level in the days preceding hospitalizations, both added to the classification of decompensation cases. In general, the features that were predictive focused on longer time frames (weeks to months) than usually considered for weight, which might be because active telemonitoring is targeted to respond to acute increases in weight and that more subtle changes are overlooked. However, it could also indicate that sharp changes in physiological signals are an uncommon presentation of acute $\mathrm{HF}$, at least in patients on telemonitoring. This analysis suggests that long-term changes are important in HF monitoring and, in particular, that HR-based features appeared to be discriminatory when a window of data of 5-12 days was considered.

The detailed analysis of the 5-day risk classifier in Fig. 5 shows that both significant increases and decreases in weight increase the risk of decompensation. Also, a falling systolic BP, after adjusting for the inherent variability in the BP, (f-6) without any change in HR add to the risk of decompensation. Possibly, this points to the body's inability to adapt the HR despite a falling BP and the associated reduction in cardiac output. Unmeasured confounders are another possibility.

The strength of this study comes from analyzing wellcharacterized hospitalizations for acute heart failure, using multi-resolution analysis based on non-invasive measurements of HR, BP, and weight at home. Further, the results of the study indicate an added benefit of incorporating measures of HR and $\mathrm{BP}$, parameters not well studied in literature, to weight-based measures for improving classification. Based on our findings of weight gain prior to $\mathrm{HFH}$, future studies may benefit from developing weight-based features that capture an uptick in weight in an otherwise downward trend.

However, there are limitations in the data used for analysis. First, only a minority of HFHs passed the data sufficiency criteria and could be analyzed. This is largely because repeat HFHs are common and occur in close proximity to one another and many within 30 days following discharge. In conjunction to algorithms, such as the one presented herein which requires, for instance, 75 event-free days prior to deployment for predictive monitoring, telemonitoring systems will need algorithms that are tailored to predict the risk of HFH in the days immediately following discharge from HFH or enrolment in to the study. This can serve as a direction for future research. Second, there exist some underlying differences between the patient populations of the two databases with regard to body mass index ( $p$-value=0.003), LVEF ( $p$-value $=0.0003)$, and serum potassium ( $\mathrm{p}$-value $=0.05)$. In the Motiva-Hull subjects, the mean values of all three characteristics are higher by $8.6 \%$, $18.5 \%$, and $3 \%$ respectively. Also, there are differences in the baseline medications between the subjects of the two databases, especially in the use of beta-blockers and vasodilators. Some of these differences were likely because the TEN-HMS database dates to the period from 2000-2002 while the Motiva-Hull database was recorded during 2008-2010. Finally, active telemonitoring acts as a confounding factor for feature/algorithm development since medical personnel may dynamically change the pharmaceutical regimen of the patient and provide lifestyle/diet advice by observing the subject's vital parameters. It is likely that the discriminatory potential of features is higher when the subject-characterization is more homogenous (for instance from the same period and from the same dataset) and if they are not actively telemonitored.

\section{CONCLUSION}

Home telemonitoring is posited to become an integral part of all chronic disease management, including HF. Algorithms that can indicate risk of decompensation by automatically analyzing daily measurements of vital signs can augment these systems and may help clinicians keep the patients stable. So far, conventional noninvasive systems have focused on weight increase alone. We have shown that although the majority of patients gained weight, some are hospitalized for worsening HF with only a small or no increase in weight. Including weight decrease and analyzing measurements of HR and BP, which are often captured in conjunction with weight, can give additional information of decompensation risk. Algorithms, such as the Naïve Bayesian approach used here, could provide a visualization of how each variable contributed to the risk estimation, thereby aiding clinicians reviewing the alert.

\section{REFERENCES}

[1] S. C. Inglis, R. a. Clark, F. a. McAlister, S. Stewart, and J. G. F. Cleland, "Which components of heart failure programmes are effective? A systematic review and meta-analysis of the outcomes of structured telephone support or telemonitoring as the primary component of chronic heart failure management in 8323 patients: Abridged Cochrane review," Eur. J. Heart Fail., vol. 13, no. 9, pp. 1028-1040, 2011.

[2] J. Polisena et al., "Home telemonitoring for congestive heart failure: a systematic review and meta-analysis.," J. Telemed. Telecare, vol. 16, no. 2, pp. 68-76, 2010.

[3] A. Conway et al., "Not all systematic reviews are systematic: A metareview of the quality of current systematic reviews and meta-analyses for remote monitoring in heart failure," J. Telemed. Telecare, vol. 19, no. 6, pp. 326-337, 2013

[4] P. Dendale et al., "Effect of a telemonitoring-facilitated collaboration between general practitioner and heart failure clinic on mortality and rehospitalization rates in severe heart failure: the TEMA-HF 1 (TElemonitoring in the MAnagement of Heart Failure) study.," Eur. J. Heart Fail., vol. 14, no. 3, pp. 333-340, 2012.

[5] F. Koehler et al., "Impact of remote telemedical management on mortality and hospitalizations in ambulatory patients with chronic heart failure: The telemedical interventional monitoring in heart failure study," Circulation, vol. 123, no. 17, pp. 1873-1880, 2011.

[6] F. Koehler et al., "Telemedicine in heart failure: Pre-specified and exploratory subgroup analyses from the TIM-HF trial," Int. J. Cardiol., vol. 161 , no. 3, pp. 143-150, 2012.

[7] S. I. Chaudhry et al., "Telemonitoring in patients with heart failure.," $N$. Engl. J. Med., vol. 363, no. 24, pp. 2301-2309, 2010. 
[8] P. Lyngå et al., "Weight monitoring in patients with severe heart failure (WISH). A randomized controlled trial," Eur. J. Heart Fail., vol. 14, no. 4, pp. 438-444, 2012.

[9] L. R. Goldberg et al., "Randomized trial of a daily electronic home monitoring system in patients with advanced heart failure: The Weight Monitoring in Heart Failure (WHARF) trial," Am. Heart J., vol. 146, no. 4, pp. 705-712, 2003.

[10] S. I. Chaudhry, Y. Wang, J. Concato, T. M. Gill, and H. M. Krumholz, "Patterns of weight change preceding hospitalization for heart failure," Circulation, vol. 116, no. 14, pp. 1549-1554, 2007.

[11] J. Lewin, M. Ledwidge, C. O'Loughlin, C. McNally, and K. McDonald, "Clinical deterioration in established heart failure: What is the value of BNP and weight gain in aiding diagnosis?," Eur. J. Heart Fail., vol. 7, no. 6, pp. 953-957, 2005.

[12] K. M. M. Ledwidge, "Evaluation of a subject's weight," Pub. No.: US 2012/0330683 A1, 2012.

[13] M. T. Ledwidge et al., "Can individualized weight monitoring using the HeartPhone algorithm improve sensitivity for clinical deterioration of heart failure?," Eur. J. Heart Fail., vol. 15, no. 4, pp. 447-455, 2013.

[14] I. Cuba Gyllensten et al., "Early Indication of Decompensated Heart Failure in Patients on Home-Telemonitoring: A Comparison of Prediction Algorithms Based on Daily Weight and Noninvasive Transthoracic Bioimpedance," JMIR Med. Inform., vol. 4, no. 1, p. e3, 2016.

[15] J. Zhang, K. M. Goode, P. E. Cuddihy, and J. G. F. Cleland, "Predicting hospitalization due to worsening heart failure using daily weight measurement: Analysis of the Trans-European Network-Home-Care Management System (TEN-HMS) study," Eur. J. Heart Fail., vol. 11, no. 4, pp. 420-427, 2009.

[16] I. Cuba Gyllensten, A. Crundall-Goode, R. M. Aarts, and K. M. Goode, "Simulated case management of home telemonitoring to assess the impact of different alert algorithms on work-load and clinical decisions," $B M C$ Med. Inform. Decis. Mak., vol. 17, p. 11, 2017.

[17] J. G. F. Cleland, A. a. Louis, A. S. Rigby, U. Janssens, and A. H. M. M. Balk, "Noninvasive home telemonitoring for patients with heart failure at high risk of recurrent admission and death: The Trans-European NetworkHome-Care Management System (TEN-HMS) study," J. Am. Coll. Cardiol., vol. 45, no. 10, pp. 1654-1664, 2005.

[18] R. Dierckx et al., "If home telemonitoring reduces mortality in heart failure, is this just due to better guideline-based treatment?," J. Telemed. Telecare, vol. 21, no. 6 pp. 331-339, 2015
[19] Y. Zhang et al., "Home telemonitoring reduces hospitalization for heart failure: a propensity-matched analysis of a routine clinical service," Eur. Heart J., vol. 34, no. suppl 1, p. 5892, Aug. 2013.

[20] G. D. Schiff, S. Fung, T. Speroff, and R. A. McNutt, "Decompensated heart failure: Symptoms, patterns of onset, and contributing factors," $\mathrm{Am}$. J. Med., vol. 114, no. 8, pp. 625-630, 2003.

[21] R. M. Oren, "Hyponatremia in congestive heart failure.," Am. J. Cardiol., vol. 95 , no. 9A, p. 2B-7B, 2005.

[22] G. Koulaouzidis, D. K. Iakovidis, and A. L. Clark, "Telemonitoring predicts in advance heart failure admissions," Int. J. Cardiol., vol. 216, pp. 78-84, 2016.

[23] G. Cotter, G. M. Felker, K. F. Adams, O. Milo-Cotter, and C. M. O'Connor, "The pathophysiology of acute heart failure--Is it all about fluid accumulation," Am. Heart J., vol. 155, no. 1, pp. 9-18, Jan. 2008.

[24] C. Fallick, P. A. Sobotka, and M. E. Dunlap, "Sympathetically Mediated Changes in Capacitance Redistribution of the Venous Reservoir as a Cause of Decompensation," Circ. Heart Fail., vol. 4, no. 5, pp. 669-675, Sep. 2011.

[25] S. von Haehling, W. Doehner, and S. D. Anker, "Nutrition, metabolism, and the complex pathophysiology of cachexia in chronic heart failure," Cardiovasc. Res., vol. 73, no. 2, pp. 298-309, Jan. 2007.

[26] A. Sandek, W. Doehner, S. D. Anker, and S. von Haehling, "Nutrition in heart failure: an update.," Curr. Opin. Clin. Nutr. Metab. Care, vol. 12, no. 4, pp. 384-391, 2009.

[27] S. Weyer et al., "Bioelectrical impedance spectroscopy as a fluid management system in heart failure," Physiol. Meas., vol. 35, no. 6, p. 917, 2014.

[28] S. J. Pocock et al., "Weight loss and mortality risk in patients with chronic heart failure in the candesartan in heart failure: assessment of reduction in mortality and morbidity (CHARM) programme," Eur. Heart J., vol. 29, no. 21, pp. 2641-2650, 2008.

[29] A. P. Ambrosy et al., "Body Weight Change During and After Hospitalization for Acute Heart Failure: Patient Characteristics, Markers of Congestion, and Outcomes: Findings From the ASCEND-HF Trial," JACC Heart Fail., vol. 5, no. 1, pp. 1-13, Jan. 2017. 